\title{
Robotic Partial Nephrectomy: Selective Arterial Clamping Technique
}

\author{
Ming Ho Edmond Wong, MBBS (HK), FRCS (Ed), FRCSEd (Urol), FCSHK, FHKAM (Surgery) \\ Department of Surgery, Urology, Queen Mary Hospital, Hong Kong, Hong Kong. \\ E-mail: drwongmingho@gmail.com
}

Chi Man Ng, MBBS (HK)

Department of Surgery, Urology, Queen Mary Hospital, Hong Kong, Hong Kong.

Ming Kwong Yiu, MBBS (HK), FRCS (Edin), Dip. Urol. (London), FHKAM (Surgery)

Department of Surgery, Urology, Queen Mary Hospital, Hong Kong, Hong Kong.

(c) Mary Ann Liebert, Inc. DOI: 10.1089/vid.2013.0053
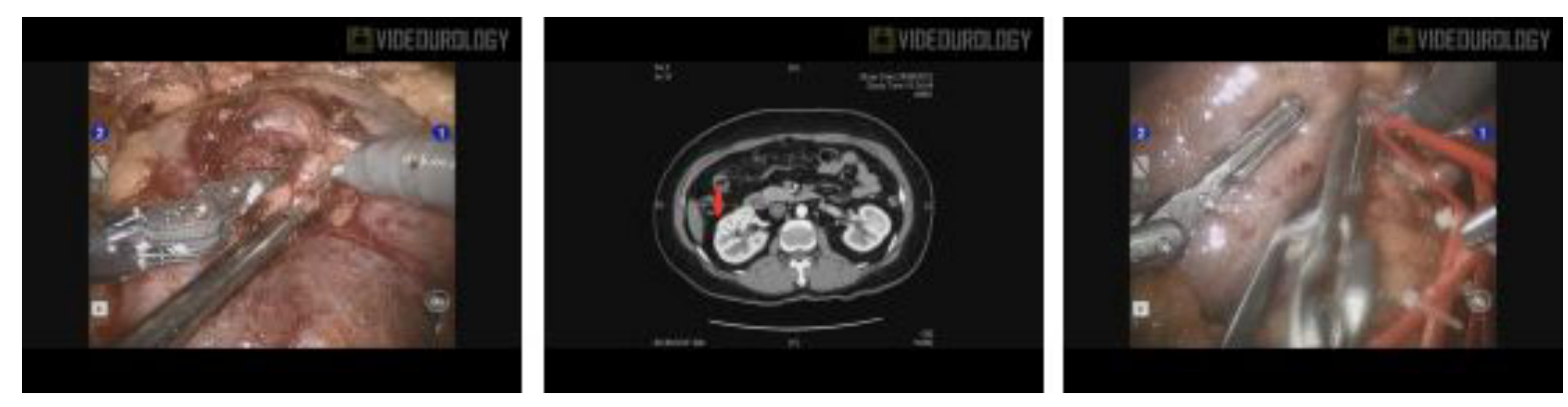

\section{Abstract}

Introduction: With widespread use of modern imaging methods, more renal cell carcinoma is diagnosed at an earlier stage. These tumors are often of lower grade and stage, and nephron-sparing surgery has become the treatment option of such small renal masses, with equivalent oncological outcome and benefit of preserved overall renal function. ${ }^{1,2}$ We present the technique of robotic partial nephrectomy with selective arterial clamping by a case illustration.

Materials and Methods: A single case of renal cell carcinoma suitable for robotic partial nephrectomy was selected for demonstration of selective arterial clamping technique. A brief introduction on the indications, selection, and options of nephron-sparing surgery was discussed. Previous results of case series on the selective arterial clamping technique on partial nephrectomy were reviewed ${ }^{3-6} \mathrm{~A}$ patient, with incidental finding of the $2 \mathrm{~cm}$ right interpolar contrast enhancing renal mass, underwent contrast CT imaging ( $2.5 \mathrm{~mm}$ cut) with vascular anatomy reconstruction as preoperative planning. After standard robotic docking, dissection of the renal hilum reveals the renal artery and segmental branches. A tumor was identified with intraoperative ultrasonography and the dissection area marked. Selective arterial clamping with utilization of the conventional bulldog clamp to demonstrate obliteration of blood supply to the tumor containing parenchyma as shown by blanching of the area and absence of Doppler signal on ultrasonography. Complete excision of the tumor with cold cut scissor and renorrhaphy with $2 / \mathrm{O} V$-lock suture in a standard fashion. Minimal blood loss was observed during dissection and no blood transfusion required.

Results: This case illustrates our early experience on a series of two cases of small renal mass undergoing robotic assisted partial nephrectomy with the selective arterial clamping technique. In both cases, selective arterial clamping is achieved by preoperative identification of the feeding vessels through computer tomography with 3D reconstruction and intraoperative ultrasonography. Without the use of near-infrared fluoresce imaging, visual identification of blanching of parenchyma is sufficient to achieve similar results. The mean clamping time is $16 \mathrm{~min}$. There was minimal blood loss and no blood transfusion required. There were no perioperative complications and no mortality. 
Conclusions: Robotic partial nephrectomy with utilization of selective arterial clamping technique is technically feasible. Utilization of advance preoperative and intraoperative imaging to demonstrate the suitable arterial branches are crucial steps in the success of the procedure.

No competing financial interests exist.

Runtime of video: 7 mins 25 secs

\section{Cite this video}

Ming Ho Edmond Wong, Chi Man Ng, Ming Kwong Yiu, Robotic Partial Nephrectomy: Selective Arterial Clamping Technique, J Endourol Part B Videourology. 2013, DOI: 10.1089/vid.2013.0053.

\section{References}

1. Ljungberg B. European Association of Urology Guideline Group. EAU guidelines on renal cell carcinoma: the 2010 update. Eur Urol 2010;58:398-406.

2. Van Poppel H. A prospective randomized EORTC intergroup phase 3 study comparing the complications of elective nephron-sparing surgery and radical nephrectomy for low-stage renal cell carcinoma. Eur Urol 2007;51:1606-1615.

3. Ficarra V. Predictors of warm ischemia time and perioperative complications in a multicenter, international series of robot-assisted partial nephrectomy. Eur Urol 2012;61:395-402.

4. Thompson RH. Every minute counts when the renal hilum is clamped during partial nephrectomy. Eur Urol 2010;58:340-345.

5. Nohara T. Modified anatrophic partial nephrectomy with selective renal segmental artery clamping to preserve renal function: a preliminary report. Int J Urol 2008;15:961-966.

6. Shao P. Laparoscopic partial nephrectomy with segmental renal artery clamping: technique and clinical outcomes. Eur Urol 2011;59:849-855.

Original Publication Date: 2013 